\title{
Transplanted embryonic spinal tissue promotes severed sciatic nerve regeneration in rats*
}

\author{
Ge Xiong ${ }^{1}$, Noriyuki Ozaki² and Yasuo Sugiura² \\ ${ }^{1}$ Department of Hand Surgery, Beijing Jishuitan Hospital, Beijing, China; and ${ }^{2}$ Department of Functional Anatomy and \\ Neuroscience, Nagoya University Graduate School of Medicine, Nagoya, Japan
}

\begin{abstract}
Summary. The effects of transplanted embryonic spinal tissue on host motor nerve regeneration and target muscle reinervation were investigated in severed sciatic nerves of rats. The electromyogram (EMG) responses and number of motor end plates (MEP) in target muscles, number of nerve axons, and retrogradely labeled motor neurons were examined in transplantation-, anastomosis without transplantation-, and naïve groups of the animals. The EMG patterns of the transplantation group returned to nearly normal at the 8th week, but those of the anastomosis group did not. MEP counts in the transplantation group were significantly higher than in the anastomosis group. The myelinated axon counts and myelin sheath thickness in the transplantation group were significantly higher than those in the anastomosis group. The number of retrogradely labeled motor neurons was significantly higher in the transplantation group. We conclude that transplanted embryonic spinal tissue can promote both host motor nerve regeneration and target muscle reinnervation.
\end{abstract}

Received July 17, 2009

* This study was supported in part by the Japan-China Sasakawa Medical Fellowship.

Address for correspondence: Prof. Yasuo Sugiura, Department of Functional Anatomy and Neuroscience, Nagoya University Graduate School of Medicine, 65 Tsurumai-cho, Showa-ku, Nagoya 466-8550, Japan

Tel: +81-52-744-2014, Fax: +81-52-744-2027

E-mail: ysugiura@med.nagoya-u.ac.jp

\section{Introduction}

Peripheral nerve injury is quite common in clinical practice. The rapid atrophy of skeletal muscle after denervation severely compromises efforts to restore muscular function. Thus, accelerating axonal regeneration to shorten the delay of reinnervation and improve functional recovery after a peripheral nerve lesion constitutes an urgent clinical demand and a major experimental challenge. It has been well documented that transplanted embryonic CNS tissues have a remarkable potential to differentiate, grow, and function, and can survive and develop in the adult peripheral nervous system (PNS) (Bernstein, 1983; Aguayo et al., 1984; Richardson and Issa, 1984; Doering, 1991). Some researchers have recently found that embryonic ventral spinal cord cells transplanted into the distal stump of an axotomized tibial nerve in adult rats could restore some degree of skeletal muscular function (Thomas et al., 2000; Klinge et al., 2001). Their EMG and morphological findings confirmed axonal regeneration of the target muscles, and their overall results excluded regeneration from the host sciatic nerves, which indicated that transplanted embryonic spinal tissues could survive and innervate host target muscles. However, whether such tissues can enhance host axonal regeneration remains unknown. In this study, we transplanted embryonic spinal tissues into severed sciatic nerves in an attempt to explore whether such transplanted tissue would improve the chance of host motor nerve regeneration and target muscle reinnervation. 


\section{Materials and Methods}

\section{Animals and surgical techniques}

Experiments were performed on 27 male Lewis rats (Japan SLC Inc., Hamamatsu) weighing 145-155 g at the beginning of the experiment. Three pregnant Lewis rats were used as the source of embryonic tissues. Animal care and procedures were approved by the Animal Experimentation Ethics Committee of Nagoya University and carried out in accordance with the Regulations for Animal Experiments of Nagoya University and the National Institute of Health Guide for the Care and Use of Laboratory Animals (NIH Publications No. 80-23) revised in 1996.

Three pregnant rats as well as 18 adult male rats were intraperitoneally anesthetized with sodium pentobarbital (30-50 mg/kg, Nembutal, Abbot Laboratories, Chicago, IL, USA). According to a previous study (Bernstein, 1983), the embryos - taken on embryonic day 12-were removed individually from the uterus and dissected in Eagle's basal medium. For spinal cord grafts, the notochord was excluded, at the cervical, thoracic, and lumbar levels, and embryonic spinal tissues were cut into small pieces of graft tissues less than $1 \mathrm{~mm}$ at most.

For the 18 host rats, both hind limbs were shaved and washed with antiseptic solution before positioning for surgery. The sciatic nerves were exposed using a gluteal muscle-splitting approach. With the aid of a surgical microscope, the bilateral sciatic nerves were carefully dissected free of the surrounding connective tissue and transected at the mid-thigh level. On the right side, the sciatic nerve was sutured end-to-end with No. 10-0 nylon microsutures immediately after the transection. On the left side, the nerve was also sutured end-to-end, but a $3-\mathrm{mm}$ incision of the epineurium was left so that some endoneurial contents could be evacuated; the embryonic spinal tissues were then grafted into the space between the proximal stump and distal stump of sciatic nerves. The incision of the epineurium was then completely closed with microsutures (Fig. 1).

The other 9 adult rats did not undergo nerve severing, and functioned as normal controls.

\section{Electromyography (EMG)}

At 4 and 8 weeks after the embryonic spinal tissue transplantation, 9 rats (including 6 nerve-severed rats and 3 normal intact rats for each stage) were anesthetized, and their sciatic nerves were exposed as previously described. Electromyograms were recorded with the RM-6000 polygraph system (Nihon Kohden, Tokyo). The evoked responses were recorded by bipolar EMG needle electrodes inserted into the belly of the gastrocnemius and anterior tibial muscle, respectively, while the ground electrode was connected to the rat's ear. The site of electrical nerve stimulation was the sciatic nerve trunk proximal to the anastomosis. Some cotton soaked in mineral oil was used to isolate the stimulating electrodes from the surrounding tissues. Nerve stimulation was performed with brief $1-\mathrm{Hz}$ pulses ( 0.1 -ms duration). The evoked EMG was recorded on a storage oscilloscope and photographed for further analysis (Fig. 2A,B). Latency was calculated as the time elapsed from stimulus onset to the first major peak of the maximum compound muscle action potentials (CMP), with amplitude measures being taken from this particular peak.

\section{Measurement of wet weight of muscles reinnervated by sciatic nerve}

One, 4 , and 8 weeks after severing the nerve, 9 rats, including 3 normal and 6 nerve-severed animals, were respectively anesthetized. The gastrocnemius and anterior tibial muscle were dissected out from bilateral hind limbs and weighed while wet (muscles of rats at the 4th and 8th week were harvested after EMG recording). The ratio of the muscle weight from a nerve-severed rat to the corresponding muscle weight from a normal intact rat, expressed as a percentage, was calculated. After weighing, the muscles were fixed with $4 \%$ paraformaldehyde in phosphate-buffered saline (PBS, pH 7.4) at $4^{\circ} \mathrm{C}$.

\section{Motor end-plate (MEP) staining and morphometric analysis}

The anterior tibial muscles taken from both normal intact and nerve-severed rats were fixed overnight with $4 \%$

\footnotetext{
Abbreviations: E12, embryo at 12 days; EMG, electromyography; MEP, motor end plates; FG, fluorogold; CNS, central nervous system; PNS, peripheral nervous system; CMP, compound muscle action potentials; SEM, standard error of the mean; ANOVA, one-way analysis of variance; GDNF, glial cell line-derived neurotrophic factor.
} 
paraformaldehyde in PBS at $4{ }^{\circ} \mathrm{C}$, after which the samples were kept in $20 \%$ sucrose in PBS for another $12 \mathrm{~h}$ at $4^{\circ} \mathrm{C}$. The tissues were then embedded in Tissue-Tek O.C.T. Compound (Sakura Finetechnical, Tokyo) and stored at $-80^{\circ} \mathrm{C}$ until sectioning. Longitudinal serial sections (20$\mu \mathrm{m}$ thick) of each muscle were obtained for staining from the midsection of the muscle belly using a cryostat (Bright Instrument Company Ltd., UK) at $-23^{\circ} \mathrm{C}$.

The protocol for staining motor end-plates was adapted from Pearson and Grose (Pearson and Grose, 1959). The incubating solution prepared just before incubation contained $8 \mathrm{~m} l$ of a $0.1 \mathrm{M}$ Tris buffer $(\mathrm{pH} \mathrm{7.2),} 3 \mathrm{ml}$ of $0.05 \mathrm{M}$ potassium ferricyanide, $3 \mathrm{~m} l$ of $0.05 \mathrm{M}$ potassium ferrocyanide, and $1 \mathrm{~m} l$ of $5 \mathrm{mg} / \mathrm{m} l 5$-bromoindoxyl acetate in ethanol. The muscle sections were washed in distilled water for $5 \mathrm{~min}$, and then immersed in the incubation solution for another $60 \mathrm{~min}$ at room temperature. After incubation, the specimens were rinsed thoroughly in distilled water before they were dehydrated, cleared, and coverslipped for observation.

The sections were observed under an Olympus AX80 microscope. Pictures of the MEP staining were captured with a digital camera attached to the microscope, and were then analyzed with the aid of Scion Image software (Scion Corporation, Frederick, MD, USA). The stained MEPs were automatically outlined by the software, thus allowing the area, signal intensity, and number of MEPs to be measured. Because of the different times of sacrifice, the individual muscle size varied considerably. To compare the results of MEP numbers among the different groups, a distribution density $(\mathrm{n} / \mathrm{mm})$ was calculated by dividing the counts of MEP by the width of the corresponding muscle belly ( $\mathrm{mm}$ ).

\section{Axon counting and myelin sheath measurement}

After the muscles were removed for weight measurements and histochemistry, the rats were perfused through the ascending aorta with about $250 \mathrm{~m} l$ of cold heparinized normal saline followed by $300 \mathrm{~m} l$ of cold $4 \%$ paraformaldehyde in PBS. Specimens were taken from the sciatic nerves $8 \mathrm{~mm}$ distal from the anastomosis site in the nerve-severed rats or at approximately the same site of the sciatic nerves in normal intact rats. The specimens were post-fixed with $1 \%$ osmium tetroxide, dehydrated in serially increasing concentrations of ethanol and QY-1 (Nisshin EM, Tokyo), and then embedded in Epon resin (Quetol 512; Nisshin EM). Each transverse section of $1 \mu \mathrm{m}$ in thickness was stained with toluidine blue and examined under a light microscope. The myelinated axons in each sciatic nerve were automatically marked and counted in digital photographs $(\times 100)$ using image analyzing software (Image-Pro Plus, Media Cybernetics, Inc., Bethesda, MD, USA). The ratio of the axon counts from a nerve-severed rat to the corresponding axon counts from a normal intact rat, expressed as a percentage, was calculated. The myelin sheath thickness of the axons was also measured in the digital photographs with the same software.

\section{Retrograde tracing and labeled motor neuron count}

At the 3 rd and 7 th week after embryonic spinal tissue transplantation, i.e., one week before being sacrificed, $50 \mu l$ of $4 \%$ hydroxy-stilbamidine (Fluoro-gold, FG) (Fluorochrome, Englewood, CO, USA) in saline was injected into the anterior tibial muscles on both sides. After sacrifice and perfusion, the spinal cord of lumbar enlargement was meticulously dissected and removed en bloc, placed in a $4 \%$ paraformaldehyde solution in PBS, and stored at $4{ }^{\circ} \mathrm{C}$ overnight for postfixation. The samples were then kept in $20 \%$ sucrose in PBS for another $12 \mathrm{~h}$ at $4{ }^{\circ} \mathrm{C}$. Following this, the tissues were embedded in TissueTek O.C.T. Compound (Sakura Finetechnical,

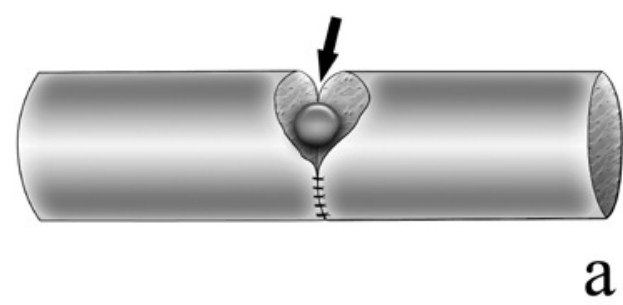

a

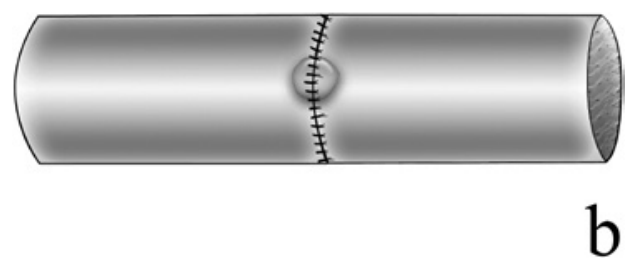

Fig. 1. Embryonic spinal tissue was transplanted into a severed sciatic nerve of a rat. a: On the left side, the sciatic nerve was sutured end-to-end, but a $3-\mathrm{mm}$ incision of the epineurium was left open so that some endoneurial contents could be evacuated; the embryonic spinal tissues were then grafted into the space between proximal stump and distal stump of sciatic nerves. b: The incision of the epineurium was then completely closed with microsutures. 

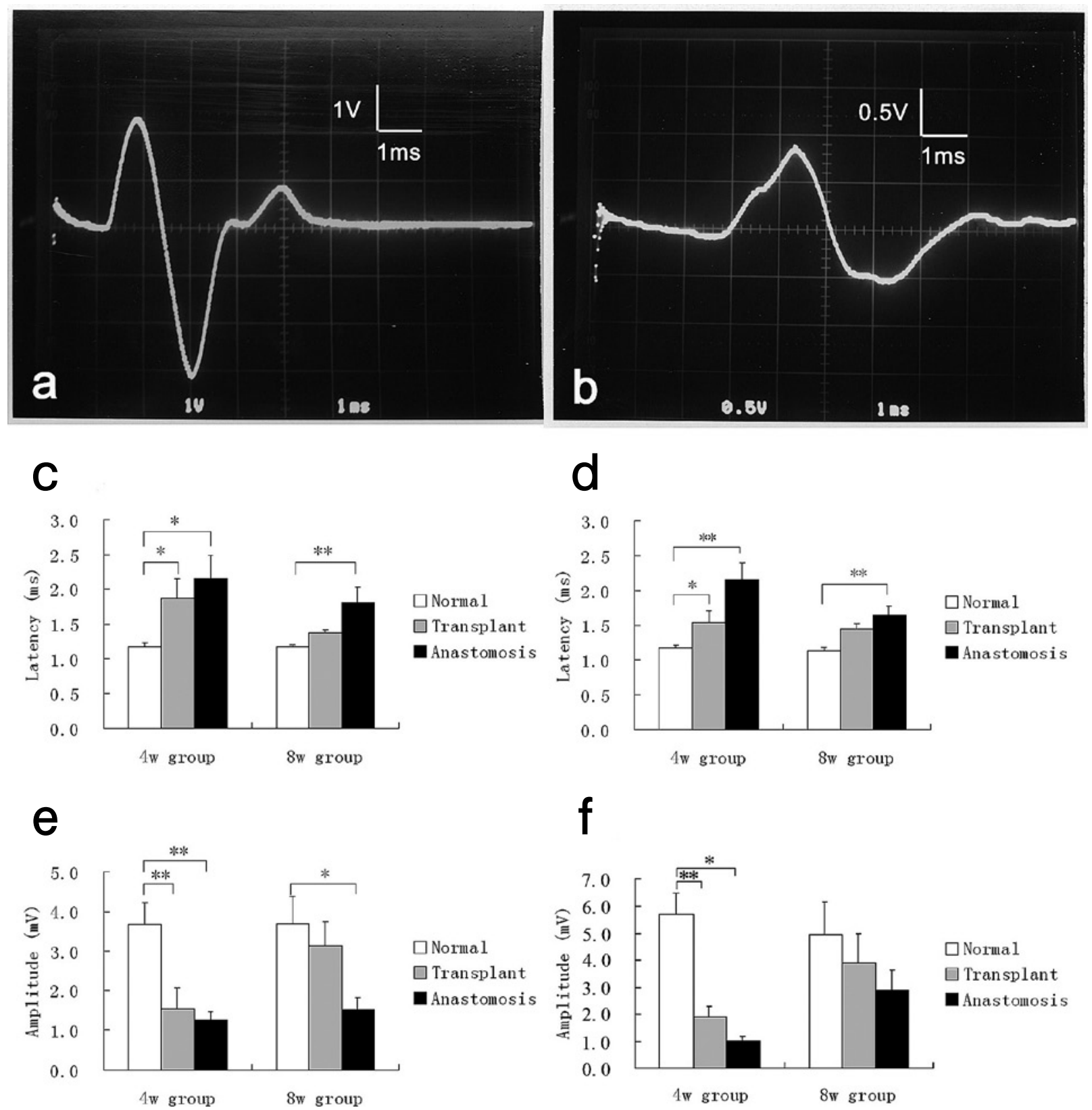

Fig. 2. Electromyography findings. a: EMG recorded from the gastrocnemius of a normal rat. b: EMG recorded from the gastrocnemius on the right regular nerve anastomosis side at the 4th week after operation. c: EMG latency of the anterior tibial muscle. At the 4th week after nerve severing, the mean latency of CMP for the anterior tibial muscle on both the left embryonic spinal tissue transplanted side and the right regular anastomosis side was longer than that of normal muscles. By the 8th week, there was a significant difference only between the right anastomosis side and the normal control group. There was no statistical difference between the left transplanted side and the normal control. d: EMG latency of gastrocnemius. At the 4th week after nerve severing, the mean latencies of CMP for the gastrocnemius on both the left transplanted side and the right anastomosis side were longer than that of the normal muscles. By the 8 th week, the only significant difference was between the right anastomosis side and the normal control (with no statistical difference between the left transplanted side and the normal control). (Continued on the next page $\nearrow$ ) 

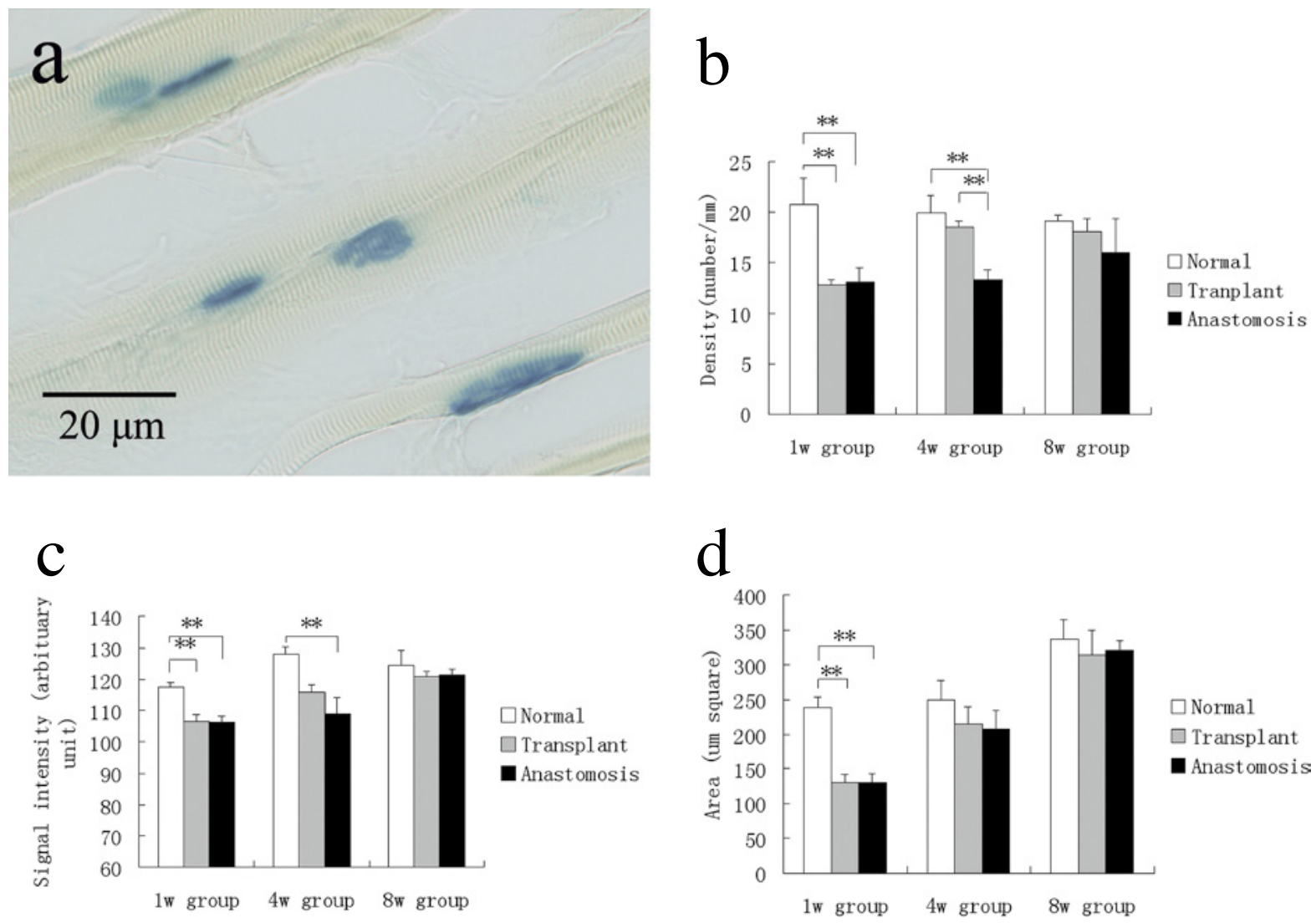

Fig. 3. Motor end-plate staining. a: MEP of the anterior tibial muscle of a normal rat stained by an esterase-histochemical technique. b: MEP distribution density of the anterior tibial muscle. At the 1st and 4th weeks after surgery, there were significant differences between the normal control and either the transplanted side or the anastomosis side. By the 8th week, there was no statistical difference among the three groups. c: MEP signal intensity of the anterior tibial muscle. At the 1 st week after surgery, there were significant differences between the normal control and either the transplanted side or the anastomosis side. At the 4th week, there was only a statistical difference between the anastomosis side and the normal control. By the 8th week, there was no statistical difference among the three groups. d: MEP area of the anterior tibial muscle. At the 1 st week, the MEP area of the anterior tibial muscle in a normal group was significantly greater than that in both the transplanted and anastomosis groups. At the 4th and 8th weeks, however, there were no differences among these three groups. $* *: \mathrm{p}<0.01$

Fig. 2. e: EMG amplitude of the anterior tibial muscle. At the 4th week postoperatively, the mean maximum CMPs for anterior tibial muscles on both the transplanted and anastomosis sides were significantly lower than that of the normal control. By the 8th week, the only statistical difference was between the anastomosis side and the normal control. f: EMG amplitude of the gastrocnemius. At the 4th week postoperatively, the mean maximum CMPs for the gastrocnemius muscles on both the transplanted and anastomosis sides were significantly lower than that of the normal control. By the 8th week, there was no statistical difference among the three groups. *: $\mathrm{p}<0.05, * *: \mathrm{p}<0.01$ 
Tokyo) and stored at $-80^{\circ} \mathrm{C}$ until sectioning. Transverse serial sections ( $15 \mu \mathrm{m}$ thick) of spinal cord were obtained using a cryostat (Bright Instrument Company, Ltd.) at $-23{ }^{\circ} \mathrm{C}$. The sections were mounted on MAS-coated glass slides (Matsunami, Tokyo), air dried for $30 \mathrm{~min}$, coverslipped in a mounting medium (Immunon, Pittsburgh, PA, USA), and stored in a black box. They were examined and photographed under an Olympus BX60 fluorescence microscope with ultraviolet filter WU. Only FG-positive motoneurons with visible nuclei were counted (ten slices for each animal).

\section{Statistical methods}

Data were expressed as the mean \pm SEM. Comparisons among the mean values of three groups were conducted using one-way analysis of variance (ANOVA) followed by a Tukey Test, and the difference between the mean values of two groups was determined by an unpaired Student's $t$-test using the SigmaStat statistical program (Systat Software, Inc., San Jose CA, USA). A $P$ value of less than 0.05 was considered statistically significant.

\section{Results}

\section{Electromyography findings}

At the 4 th week after severing the nerve, the mean latencies of CMP for the anterior tibial muscle and gastrocnemius on the left embryonic spinal tissuetransplanted side of nerve-severed rats $(n=6)$ were $1.9 \pm 0.3 \mathrm{~ms}$ and $1.5 \pm 0.2 \mathrm{~ms}$, respectively, while those on the right regular nerve anastomosis side $(n=6)$ were $2.2 \pm 0.3 \mathrm{~ms}$ and $2.2 \pm 0.6 \mathrm{~ms}$, and those in normal intact rats (normal control, 6 sciatic nerves in 3 rats, $n=6$ ) were $1.2 \pm 0.1 \mathrm{~ms}$ and $1.2 \pm 0.1 \mathrm{~ms}$ (Fig. 2C, D). The mean maximum CMP amplitudes for the anterior tibial muscle and gastrocnemius on the transplanted side $(n=6)$ of nerve-severed rats were $1.6 \pm 0.5 \mathrm{mV}$ and $1.9 \pm 0.4$ $\mathrm{mV}$, respectively, while those on the anastomosis side $(\mathrm{n}=6)$ were $1.3 \pm 0.2 \mathrm{mV}$ and $1.0 \pm 0.2 \mathrm{mV}$, and those in the normal control group $(n=6)$ were $3.7 \pm 0.6 \mathrm{mV}$ and $5.7 \pm 0.8 \mathrm{mV}$, respectively (Fig. 2E, F). There were significant differences in both the mean latency and mean maximum CMP amplitude between either side of nervesevered rats and normal rats. Although trends toward shorter latency and higher amplitude were observed in the transplanted side compared with the anastomosis side, no significant difference was found between the two sides.

At the 8th week after surgery, the mean latencies for the anterior tibial muscle and gastrocnemius on the transplanted side of nerve-severed rats $(n=6)$ were $1.4 \pm 0.1 \mathrm{~ms}$ and $1.4 \pm 0.1 \mathrm{~ms}$, respectively, while those on the anastomosis side $(\mathrm{n}=6)$ were $1.8 \pm 0.2 \mathrm{~ms}$ and $1.7 \pm 0.1 \mathrm{~ms}$, and those in the normal control group $(\mathrm{n}=6)$ were $1.2 \pm 0.03 \mathrm{~ms}$ and $1.1 \pm 0.04 \mathrm{~ms}$, respectively (Fig. 2C, D). The mean maximum CMP amplitudes for the anterior tibial muscle and gastrocnemius on the transplanted side $(n=6)$ of nerve-severed rats were $3.1 \pm 0.6 \mathrm{mV}$ and $3.9 \pm 1.1 \mathrm{mV}$, respectively, while those on the anastomosis side $(n=6)$ were $1.5 \pm 0.3 \mathrm{mV}$ and $2.9 \pm 0.8 \mathrm{mV}$, and those in the normal control group $(\mathrm{n}=6)$ were $3.7 \pm 0.7 \mathrm{mV}$ and $5.0 \pm 1.2 \mathrm{mV}$, respectively (Fig. 2E, F). For all measured parameters, there were still significant differences between the anastomosis side of nerve-severed and normal control rats, except for the mean maximum amplitude of the gastrocnemius. No significant differences, however, were found between the transplanted side and the normal control rats, suggesting a faster recovery of the transplanted side compared with the anastomosis one (Fig. 2A, B).

The EMG data from the 4th and 8th weeks also showed that the recovery of the gastrocnemius was faster and better than that of the anterior tibial muscle on both sides of nerve-severed rats.

Table 1. Wet weights of the gastrocnemius and anterior tibial muscle

\begin{tabular}{cccccc}
\hline & \multicolumn{2}{c}{$\begin{array}{c}\text { Gastrocnemius } \\
(\% \text { vs. normal control) }\end{array}$} & & \multicolumn{2}{c}{$\begin{array}{c}\text { Anterior tibial muscle } \\
(\% \text { vs. normal control })\end{array}$} \\
\cline { 2 - 3 } \cline { 5 - 6 } \cline { 5 - 6 } 1w Group & $57.5 \pm 3.0$ & $56.8 \pm 3.6$ & & $65.3 \pm 2.4$ & $64.6 \pm 1.5$ \\
4w Group & $73.1 \pm 5.0$ & $67.2 \pm 5.4$ & & $67.6 \pm 7.7$ & $66.6 \pm 2.8$ \\
8w Group & $72.3 \pm 9.3$ & $68.7 \pm 7.2$ & & $75.2 \pm 0.7$ & $70.6 \pm 5.3$ \\
\hline
\end{tabular}

Wet weights of muscles are presented as percentages versus the corresponding muscles of normal rats at the same age. 


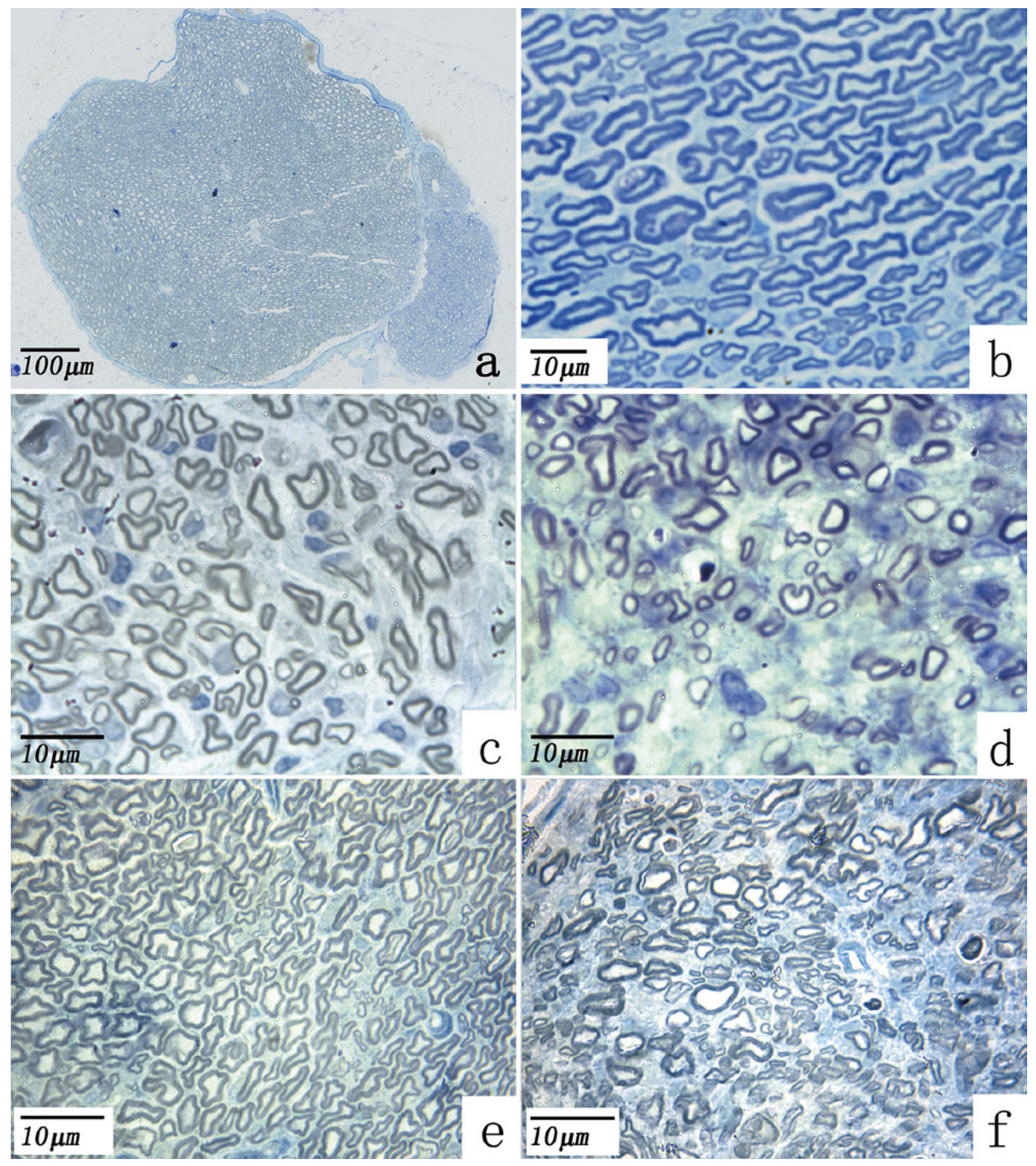

Fig. 4. Toluidine blue staining of axons in the sciatic nerve. a: Sciatic nerve of normal rat. b: Sciatic nerve of a normal rat at high magnification. c: Sciatic nerve on the left embryonic spinal tissue transplanted side of nerve-severed rats at the 4th week after surgery. d: Sciatic nerve on the right regular nerve anastomosis side of nerve-severed rats at the 4th week postoperatively. e: Sciatic nerve on the left embryonic spinal tissue transplanted side of nerve-severed rats at the 8th week after surgery. f: Sciatic nerve on the right regular nerve anastomosis side of nerve-severed rats at the 8th postoperative week. 


\section{Wet weight of gastrocnemius and anterior tibial muscle}

The wet weights of the gastrocnemius and anterior tibial muscle were presented as percentages versus the corresponding muscles of normal control rats of the same age (Table 1). In this way, the data from different time points could be compared so that the speed of muscle recovery could be evaluated.

The results of measurements showed that, at different times, and for different muscles, the mean value of muscle weight on the embryonic spinal tissue transplanted side was always higher than that on the regular nerve
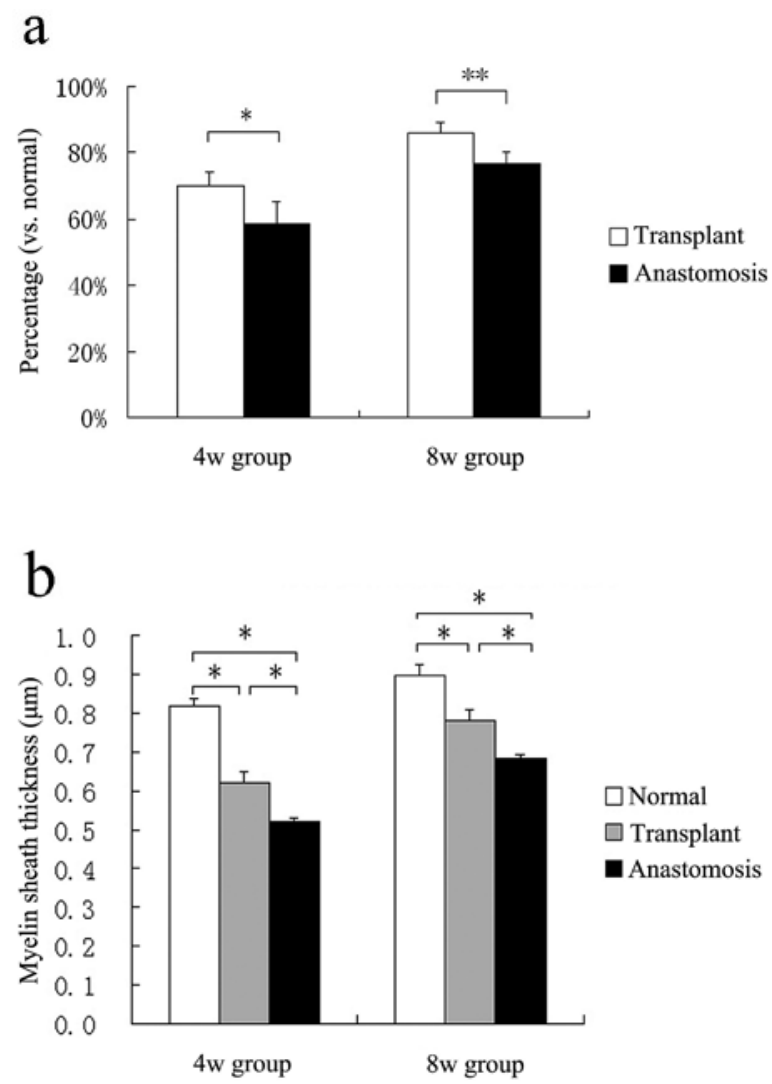

Fig. 5. Myelinated axon analysis. a: Myelinated axon counts. At the 4 th and 8 th week, the myelinated axon counts in the sciatic nerve of the transplanted group were significantly greater than that in the anastomosis group. b: Myelin sheath thickness. At both the 4th and 8th week after nerve severing, there were significant differences in myelin sheath thickness between each pair of the three groups. $*: \mathrm{p}<0.05, * *: \mathrm{p}<0.01$. anastomosis side, although the difference was not significant. As to the speed of muscle recovery, it seemed that the gastrocnemius recovered faster than anterior tibial muscle; however, after 4 weeks, the recovery slowed down, and the rate of its weight increase was almost the same as that in a normal rat, so that the muscle weight at 8 weeks was almost the same as that at 4 weeks. The fastest recovery of the anterior tibial muscle occurred between the 4 th and 8 th weeks.

\section{Motor end-plate counts}

For convenient comparisons, the MEP counts were presented as a distribution density. At the 1st week after nerve severing, the mean MEP distribution density of the anterior tibial muscle on the embryonic spinal tissue transplanted side was $12.8 \pm 0.5 \mathrm{~mm}$, against $13.1 \pm 1.4$ $\mathrm{mm}$ on the regular nerve anastomosis side, and $20.8 \pm 2.6$ $\mathrm{mm}$ in the normal control (Fig. 3A, B). Although there were significant differences between normal controls and either side of the nerve-severed muscles, there was no significant difference between the transplanted and anastomosis sides.

At the 4th week, the mean MEP distribution density on the transplanted side increased to $18.6 \pm 0.5 \mathrm{~mm}$, while that on the anastomosis side was still $13.3 \pm 0.9 \mathrm{~mm}$ (Fig. 3B). The mean distribution density of the normal control was $19.9 \pm 1.7 \mathrm{~mm}$. There were significant differences not only between the normal control and the anastomosis side, but also between the transplanted and anastomosis sides.

At the 8th week, although the mean MEP distribution density on the transplanted side $(18.1 \pm 1.2 \mathrm{~mm})$ was still higher than that on the anastomosis side $(16.0 \pm 3.3 \mathrm{~mm})$, there was no significant difference among them and the normal control (19.1 $\pm 0.6 \mathrm{~mm})$ (Fig. 3B).

\section{MEP morphometric analysis}

Compared with the normal control (117.4 \pm 1.6$)$, the mean MEP signal intensity of the anterior tibial muscle decreased significantly within the 1 st week for both sides of nerve-severed rats $(106.5 \pm 2.0$ on the transplanted side, and $106.5 \pm 1.5$ on the anastomosis side) (Fig. 3C). At the 4th week postoperatively, however, since the mean MEP signal intensity clearly increased on the transplanted side, there was no longer any significant difference between the transplanted side $(115.7 \pm 2.6)$ and normal control $(127.9 \pm 2.6)$, while the difference between the anastomosis side $(108.7 \pm 5.6)$ and the normal control remained significant (Fig. 3C). At the 8th week, we could find no significant difference among the three groups (Fig. $3 C)$. 

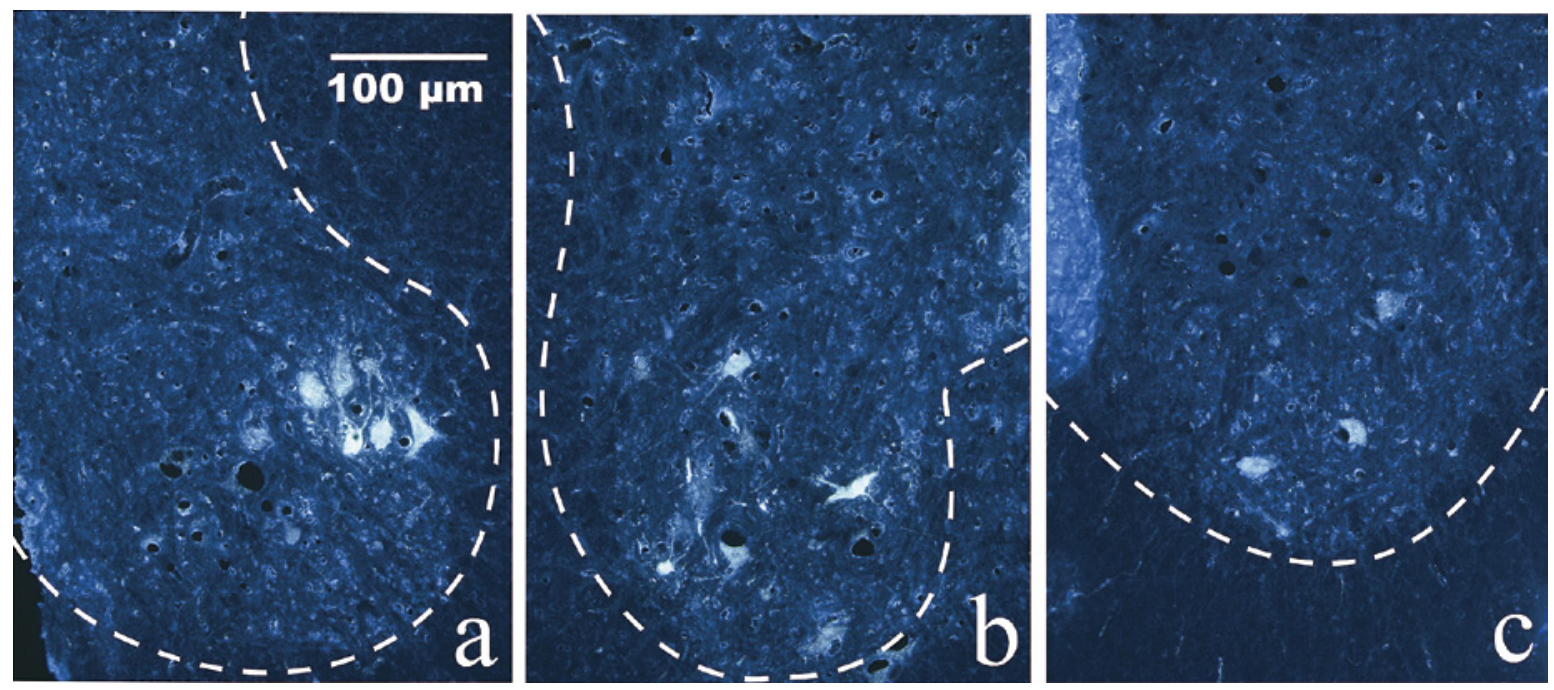

Fig. 6. Fluoro-gold labeled motor neurons. Those in a normal rat (a), on the left embryonic spinal tissue transplanted side of nerve-severed rats at the 4th week (b), and on the right regular nerve anastomosis side of nerve severed rats at the 4th week (c).

Since there was an obvious collapse of MEPs following the nerve transection, the measurement data showed a sharp decrease in the mean MEP area on both sides $\left(130.9 \pm 12.0 \mu \mathrm{m}^{2}\right.$ for the transplanted side and $130.7 \pm 13.5 \mu \mathrm{m}^{2}$ for the anastomosis side) at the $1 \mathrm{st}$ week after nerve severing, which was just short of being only one-half the normal size of MEP $\left(238.5 \pm 14.7 \mu \mathrm{m}^{2}\right)$ (Fig. 3D). Interestingly, at the 4th week, although there were still some differences among the groups, they were not significant (Fig. 3D). The same results were found at the 8th week (Fig. 3D), indicating that once the MEP recovered, it grew to its normal size.

\section{Myelinated axon counts}

One week after sciatic nerve transection, most of the myelinated axons degenerated on both sides of the operated rats, but then regenerated by the 4 th week postoperatively (Fig. 4A-D). On the embryonic spinal tissue transplanted side, the percentage of myelinated axon counts versus those of normal controls at matching distances was $70.2 \pm 3.5 \%$, while that percentage on the regular nerve anastomosis side was $58.4 \pm 6.7 \%$. A significant difference was evident between the transplanted and anastomosis sides $(\mathrm{p}<0.05)$ (Fig. 5A). This indicated that, 4 weeks after embryonic spinal tissue transplantation, there were about $20 \%$ more myelinated axons passing through a point $8 \mathrm{~mm}$ distal to the suture

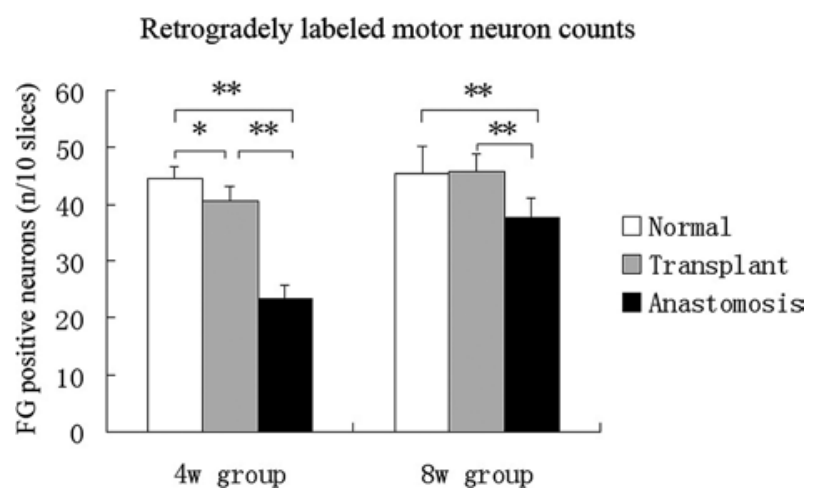

Fig. 7. Retrogradely labeled motor neuron counts. At the 4th week, significantly more motor neurons were retrogradely labeled with fluorogold in the transplanted side compared with the nerve anastomosis side. There were also significant differences between either side of the nerve-severed and normal control rats. By the 8 th week, the difference between the transplanted side and the normal control had disappeared, although there were still significant differences between the transplanted and anastomosis sides, and between the anastomosis side and normal controls. *: $\mathrm{p}<0.05, * *$ : $\mathrm{p}<0.01$. 
than were detected on the contralateral side repaired with regular nerve anastomosis without embryonic spinal tissue transplantation. By the 8th week, the percentages on both the transplanted and anastomosis sides increased up to $86.1 \pm 2.8 \%$ and $77.0 \pm 2.2 \%$, respectively, leaving a still significant difference between the two sides $(\mathrm{p}<0.01)$ (Fig. 4E, F, 5A). This demonstrated that the advantage of axon regeneration on the transplanted side persisted for at least 8 weeks (Fig. 5A).

\section{Myelin sheath thickness}

At the 4th week after nerve severing, the mean myelin sheath thickness was $0.62 \pm 0.03 \mu \mathrm{m}$ on the transplanted side and $0.52 \pm 0.01 \mu \mathrm{m}$ on the anastomosis side, while that of the normal control was $0.82 \pm 0.02 \mu \mathrm{m}$. There were significant differences between each pair of the three groups ( $<<0.05$ ) (Fig. 4C, D, 5B). By the 8th week, the thickness had increased to $0.78 \pm 0.03 \mu \mathrm{m}$ on the transplanted side and $0.68 \pm 0.01 \mu \mathrm{m}$ on the anastomosis side, while that of the normal control was $0.90 \pm 0.03 \mu \mathrm{m}$, confirming that significant differences still existed between each pair of the three groups $(\mathrm{p}<0.05)$ (Fig. 4E, F, 5B).

\section{FG labeled motor neuron counts}

At the 4 th week after nerve severing, significantly more motor neurons were retrogradely labeled with fluorogold on the embryonic spinal tissue transplanted side compared with the regular nerve anastomosis side of the nervesevered rats $(p<0.01)$. There were also significant differences between either side of the nerve-severed rats and the normal control rats (Fig. 6,7).

By the 8th week after nerve severing, the difference between the transplanted side and the normal control had disappeared, although there were still significant differences between the transplanted and anastomosis sides of the nerve-severed rats $(\mathrm{p}<0.01)$ as well as between the anastomosis side and the normal control $(\mathrm{p}<$ 0.01) (Fig. 7).

\section{Discussion}

The effects of transplanted embryonic spinal tissue on host motor nerve regeneration and target muscle reinervation were investigated in severed sciatic nerves of rats. From our physiological and morphological data, we concluded that transplanted embryonic spinal tissue could promote both host motor nerve regeneration and target muscle reinervation.

Embryonic spinal tissues contain not only neural progenitor cells-which are able to differentiate, grow and function-but also many neuroglia cells that support, nourish, and regulate the development of neurons (Grumbles et al., 2002; Grant et al., 2005). Although previous studies showed that the embryonic tissues transplanted from CNS could survive in PNS and even extend their axons to the target muscles (Richardson and Issa, 1984; Doering et al.,1991; Thomas et al., 2000; Klinge et al., 2001), such a level of innervation was insufficient for functional recovery and could not be controlled by the CNS. The main purpose of embryonic tissue transplantation in previous studies was to prevent skeletal muscle atrophy after its denervation. In contrast, the present research results showed that transplanted embryonic spinal tissues could significantly enhance the regeneration of "host" motor axons in sciatic nerves and could also expedite the reinnervation of the target muscles. We found nerve recovery to be faster and more complete in both its morphometric and electrophysiological aspects.

Recently, neural stem cell transplantation has been thoroughly investigated, not only for the CNS (Fillmore et al., 2005; Okano et al., 2005; Wong et al., 2005) but also the PNS (MacDonald et al., 2003). In the CNS, transplanted neurons may replace some destroyed host neurons and therefore restore the impaired functions. However, only a very few transplanted neurons can survive and function in PNS, according to our observations and those of other researchers (Richardson and Issa, 1984; MacDonald et al., 2003). In our experiment, we found that the embryonic graft barely survived more than 2 weeks after the transplantation. Thus, we consider it impractical to expect transplanted neurons in PNS to fully function over a long period like host neurons in the spinal cord. The reason we chose to work with embryonic spinal tissue instead of a group of neurons as a graft was to make better use of the functions of both neurons and neuroglia. The neuroglia cells can produce several neurotrophic factors, including the glial cell line-derived neurotrophic factor (GDNF) (Coull et al., 2005; Hashimoto et al., 2005; Toyomoto et al., $2005)$ that belongs to the transforming growth factor- $\beta$ superfamily (Lin et al., 1993; Henderson et al., 1994). It has a potent trophic effect not only on CNS but also on some peripheral sensory neurons (Matheson et al., 1997; Bar et al., 1998), sympathetic neurons (Buj-Bello et al., 1995; Trupp et al., 1995; Schober et al., 1999), and spinal motor neurons (Henderson et al., 1994; Yan et al., 1995; Bar et al., 1998). Such neurotrophic factors may keep the neurons in graft tissue surviving longer and functioning 
better, as well as preventing the host neurons from apoptosis after axotomy. Recent research has confirmed the expression of the GDNF family receptor in peripheral nerve Schwann cells (Hase et al., 2005). GDNF can stimulate the migration of Schwann cells and enhance myelination (Iwase et al., 2005). Our experiments also showed a similar result: there were more regenerated myelinated axons and a thicker myelin sheath formation at 4 and 8 weeks after the transplantation of embryonic spinal tissues compared with standard nerve anastomosis.

Since the MEP is a connection between the nerve and muscle, its functional recovery is absolutely essential for the restoration of motor function. Our experimental results were rather intriguing for what they showed about the several indices of the MEP. The data revealed that the size of the MEP was almost normal for rats operated at the 4th and 8th weeks, suggesting that once the MEP regenerates, it grows back to its normal size. The distribution density of the MEP might indicate the innervation by regenerated axons, while the MEP functional status might be represented by its staining intensity. The more enzymes were stored in the MEP, the darker it stained. At the 4th week, the difference was significant for the MEP counting between both sides of operated rats, which was consistent with the result of axon counting, but not quite significant enough for the staining intensity of MEP. That indicates that recovery of the MEP signal intensity is slower than the recovery of the distribution density, i.e., functional recovery follows morphological recovery.

From the EMG results, we found that the recovery of the gastrocnemius was faster than that of the anterior tibial muscle. A similar situation has been seen in patients with sciatic nerve injuries. Thus at the 8 th week, though there was no significant differences in amplitude among the three groups for gastrocnemius, a significant difference remained for the anterior tibial muscle between normal controls and the regular nerve anastomosis side of nerve-severed rats.

In our experiments, we observed no significant differences in muscle wet weights between the transplanted side and the anastomosis side of nervesevered rats. This may imply that the embryonic spinal tissue is not capable of preventing muscular atrophy.

In summary, the transplantation of embryonic spinal tissue to a severed sciatic nerve can significantly enhance the recovery of injured motor nerves and expedite the reinnervation of the target muscles, although the underlying mechanism is still not fully understood. Future research into the interactions between a CNS graft and host PNS will greatly enhance our knowledge of the mechanism of peripheral nerve regeneration and promote the repair of peripheral nerve injury.

\section{References}

Aguayo AJ, Bjorklund A, Stenevi U, Carlstedt T: Fetal mesencephalic neurons survive and extend long axons across peripheral nervous system grafts inserted into the adult rat striatum. Neurosci Lett 45: 53-58 (1984).

Bar KJ, Saldanha GJ, Kennedy AJ, Facer P, Birch R, Carlstedt T, Anand P: GDNF and its receptor component Ret in injured human nerves and dorsal root ganglia. Neuroreport 9: 43-47 (1998).

Bernstein JJ: Viability, growth, and maturation of fetal brain and spinal cord in the sciatic nerve of adult rat. $J$ Neurosci Res 10: 343-350 (1983).

Buj-Bello A, Buchman VL, Horton A, Rosenthal A, Davies AM: GDNF is an age-specific survival factor for sensory and autonomic neurons. Neuron 15: 821828 (1995).

Coull JA, Beggs S, Boudreau D, Boivin D, Tsuda M, Inoue K, Gravel C, Salter MW, De Koninck Y: BDNF from microglia causes the shift in neuronal anion gradient underlying neuropathic pain. Nature 438: 1017-1021 (2005).

Doering LC: Transplantation of fetal CNS tissue into the peripheral nervous system: a model to study aberrant changes in the neuronal cytoskeleton. J Neural Transplant Plast 2: 193-205 (1991).

Fillmore HL, Holloway KL, Gillies GT: Cell replacement efforts to repair neuronal injury: a potential paradigm for the treatment of Parkinson's disease. NeuroRehabilitation 20: 233-242 (2005).

Grant KA, Raible DW, Piotrowski T: Regulation of latent sensory hair cell precursors by glia in the zebrafish lateral line. Neuron 45: 69-80 (2005).

Grumbles RM, Wood P, Rudinsky M, Gomez AM, Thomas CK: Muscle reinnervation with delayed or immediate transplant of embryonic ventral spinal cord cells into adult rat peripheral nerve. Cell Transplant 11: 241-250 (2002).

Hase A, Saito F, Yamada H, Arai K, Shimizu T, Matsumura K: Characterization of glial cell linederived neurotrophic factor family receptor alpha-1 in peripheral n erve Schwann cells. J Neurochem 95: 537543 (2005).

Hashimoto M, Ito T, Fukumitsu H, Nomoto H, Furukawa Y, Furukawa S: Stimulation of production of glial cell line-derived neurotrophic factor and nitric oxide by lipopolysaccharide with different dose-responsiveness in cultured rat macrophages. Biomed Res 26: 223-229 (2005). 
Henderson CE, Phillips HS, Pollock RA, Davies AM, Lemeulle C, Armanini M, Simmons L, Moffet B, Vandlen RA, Simpson LC, et al: GDNF: a potent survival factor for motoneurons present in peripheral nerve and muscle. Science 266: 1062-1064 (1994).

Iwase T, Jung CG, Bae H, Zhang M, Soliven B: Glial cell line-derived neurotrophic factor-induced signaling in Schwann cells. J Neurochem 94: 1488-1499 (2005).

Klinge PM, Groos S, Wewetzer K, Haastert K, Rosahl S, Vafa MA, Hosseini H, Samii M, Brinker T: Regeneration of a transected peripheral nerve by transplantation of spinal cord encapsulated in a vein. Neuroreport 12: 1271-1275 (2001).

Lin LF, Doherty DH, Lile JD, Bektesh S, Collins F: GDNF: a glial cell line-derived neurotrophic factor for midbrain dopaminergic neurons. Science 260: 11301132 (1993).

MacDonald SC, Fleetwood IG, Hochman S, Dodd JG, Cheng GK, Jordan LM, Brownstone RM: Functional motor neurons differentiating from mouse multipotent spinal cord precursor cells in culture and after transplantation into transected sciatic nerve. $J$ Neurosurg 98: 1094-1103 (2003).

Matheson CR, Carnahan J, Urich JL, Bocangel D, Zhang TJ, Yan Q: Glial cell line-derived neurotrophic factor (GDNF) is a neurotrophic factor for sensory neurons: comparison with the effects of the neurotrophins. $J$ Neurobiol 32: 22-32 (1997).

Okano H, Okada S, Nakamura M, Toyama Y: Neural stem cells and regeneration of injured spinal cord. Kidney Int 68: 1927-1931 (2005).

Pearson B, Grose F: Further histochemical studies of esterases by 5-bromoindoxyl acetate. AMA Arch Path 67: 98-106 (1959).
Richardson PM, Issa VM: Transplantation of embryonic spinal and cerebral tissue to sciatic nerves of adult rats. Brain Res 298: 146-148 (1984).

Schober A, Hertel R, Arumae U, Farkas L, Jaszai J, Krieglstein K, Saarma M, Unsicker K: Glial cell line-derived neurotrophic factor rescues targetdeprived sympathetic spinal cord neurons but requires transforming growth factor-beta as cofactor in vivo. $J$ Neurosci 19: 2008-2015 (1999).

Thomas CK, Erb DE, Grumbles RM, Bunge RP: Embryonic cord transplants in peripheral nerve restore skeletal muscle function. J Neurophysiol 84: 591-595 (2000).

Toyomoto M, Inoue S, Ohta K, Kuno S, Ohta M, Hayashi $\mathrm{K}$, Ikeda $\mathrm{K}$ : Production of NGF, BDNF and GDNF in mouse astrocyte cultures is strongly enhanced by a cerebral vasodilator, ifenprodil. Neurosci Lett 379: 185189 (2005).

Trupp M, Ryden M, Jornvall H, Funakoshi H, Timmusk T, Arenas E, Ibáñez CF: Peripheral expression and biological activities of GDNF, a new neurotrophic factor for avian and mammalian peripheral neurons. $J$ Cell Biol 130: 137-148 (1995).

Wong AM, Hodges H, Horsburgh K: Neural stem cell grafts reduce the extent of neuronal damage in a mouse model of global ischaemia. Brain Res 1063: 140-150 (2005).

Yan Q, Matheson C, Lopez OT: In vivo neurotrophic effects of GDNF on neonatal and adult facial motor neurons. Nature 373: 341-344 (1995). 